\title{
The Spectra of Very Luminous IRAS Galaxies ${ }^{1}$
}

Hong $\mathrm{Wu}$ and Zhenlong Zou

Beijing Astronomical Observatory, Chinese Academy of Sciences, China

Xiaoyang Xia

Dept. of Physics, Tianjin Normal University, China

Zugan Deng

Dept. of Physics, Graduate School, Chinese Academy of Sciences, China

\begin{abstract}
We discuss new optical spectra and DSS images of a complete sample of very luminous IRAS galaxies. The statistics of classification of spectral types and interactions show that interaction can account for the nuclear activity (AGN and starburst) of ultraluminous infrared galaxies.
\end{abstract}

\section{Sample Selection}

The very luminous IRAS galaxies selected are from the 1.936 Jy Redshift Survey (Strauss et al. 1992). The selection criteria are (1) declination $\delta \geq 0^{\circ}$, (2) IR luminosity $\log \left(L_{\mathrm{IR}} / L_{\odot}\right) \geq 11.5$ (assuming $H_{0}=50 \mathrm{~km} \mathrm{~s}^{-1} \mathrm{Mpc}^{-1}$ and $q_{0}=0.5$ ), and (3) and apparent magnitude $m_{\text {Zwicky }} \leq 15.5 \mathrm{mag}$. These criteria yield a sample of 73 sources from the $1.936 \mathrm{Jy}$ catalog.

\section{Results}

We spent nearly two years, from 1994 April 15 to 1996 February 15, obtaining spectra with the Zeiss universal spectrograph and Tek $512 \times 512$ and $1024 \times 1024$ CCD cameras on the $2.16-\mathrm{m}$ telescope at Xinglong Station, BAO. We employed a 195 lines $\mathrm{mm}^{-1}$ grating giving $10 \AA$ (2 pixels) resolution, and coverage over the range $3700-7000 \AA$. We obtained 123 spectra of galaxies, including 73 infrared sources companions and ten other infrared galaxies from the $1.93 \mathrm{Jy}$ catalog. The data were processed in a standard way with the IRAF package.

Several dereddened emission-line ratios were used to classify all the galaxies, following the guidelines of Veilluex \& Osterbrock (1987).

Images from the Digital Sky Survey (DSS) were also examined. Galaxies were also sorted by interaction class, following Lawrence (1989), which includes

${ }^{1}$ This work is supported by the National Natural Science Foundation of China. 
seven classes (0-6). There are seven confirmed groups in our sample, in which we have identified at least three companions.

In the $\alpha(100,60)$ versus $\alpha(60,25)$ diagram, all the Seyfert 1 s are above $85 \mathrm{~K}$ and only one is in the upper-left region of power-law regime, which is different from optical selected Seyfert 1s, which are mostly between $70 \mathrm{~K}$ and $90 \mathrm{~K}$.

The relationship between infrared luminosities and projected separations shows that infrared luminosity increases as the separation decreases. We find a separation of $10 \mathrm{kpc}\left(H_{0}=50 \mathrm{~km} \mathrm{~s}^{-1} \mathrm{Mpc}^{-1}\right)$ for enhancement of infrared luminosity. The relationship between projected separations and radial relative velocities or angular momemtum show that dynamical friction plays a important role even when two interacting galaxies are at large separations. This indicates a large transfer of angular momemtum occurs in the merger process.

\section{Conclusions}

1. About half $(51 \%$, or $37 / 73)$ of very luminous IRAS galaxies have AGNlike spectra (Seyfert 1s, Seyfert 2s, LINERs and mixtures of LINERs and $\mathrm{H}$ II galaxies). This fraction goes up to $73 \%$ for the subsample with $\log \left(L_{\mathrm{IR}} / L_{\odot}\right) \geq 12.0$. These fractions are somewhat higher than given by Veilleux et al. (1995).

2. Infrared-luminous Seyfert 1 galaxies show different properties from optically selected Seyfert $1 \mathrm{~s}$.

3. We find that $56 \%$ of ultraluminous infrared galaxies fall into the class of strongly interacting or merging systems, as do nearly all (91\%) highinfrared luminosity, $\log \left(L_{I R} / L_{\odot}\right) \geq 12.0$, sources. This result agrees with Sanders et al. (1988) and is larger than reported by Zou et al. (1991).

4. Strong interaction accounts for high-infrared luminosity in our sample.

\section{References}

Lawrence, A., 1989, MNRAS, 240, 329,

Sanders, D. B., et al. 1988, ApJ, 325, 74.

Sekiguchi, K. 1987, ApJ, 316, 145.

Strauss, M. A., et al. 1992, ApJS, 83, 29.

Veilleux, S., \& Osterbrock, D.E. 1987, ApJS, 63, 295.

Veilluex, S., et al. 1995, ApJS, 98, 171.

Zou, Z. L., et al. 1991, MNRAS, 252, 593. 